\title{
ВЛИЯНИЕ ДЕФОРМАЦИИ РАСТУЩИХ В ЭЛЕКТРОМАГНИТНОМ ПОЛЕ КАПЕЛЬ НА ИХ РАССЕИВАЮЩИЕ СВОЙСТВА
}

Введение. Известно, что в условиях свободного падения в облаках и осадках капли десоормируются и принимают различные формы. Экспериментальные работы, выполненные в аэродинамической трубе, показывают, что капли под влиянием электромагнитного поля еще больше десормируются. Такие работы позволяют более точно оценить размеры, форму и ориентацию капель, что находит применение при исследовании их рассеивающих свойств (поперечных сечений обратного рассеяния).

Материалы и методы исследования. Для определения фактора формы капель, растущих в грозоградовых облаках, были использованы результаты численного моделирования, лабораторных исследований в аэродинамической трубе и натурных экспериментов с поляриметром. В случае сферических капель расчеты характеристик рассеяния были проведены по Ми. Для деформированных капель использовался метод разделения переменных, модифицированный для частиц формы произвольного сфероида.

Результаты исследований и их обсуждение. Проведены расчеты поперечных сечений обратного рассеяния деформированных капель, растущих под влиянием горизонтального электрического поля. Рассмотрены капли с различными степенями десрормации. Для сравнительного анализа представлены расчеты поперечного сечения обратного рассеяния сферических капель. Показано, что при увеличении степени деформации растущих в электрическом поле капель, значения поперечных сечений обратного рассеяния более существенно отклоняются от аналогичных значений для эквивалентных сфер.

Выводы. $\quad$ Полученные результаты могут быть полезны при разработке методов радиолокационного исследования грозоградовых облаков.

Ключевые слова: теория рассеяния, поперечное сечения обратного рассеяния, степень дефрормации капель, разбрызгивание капель, грозоградовые облака. 
Sozaeva L.T., $\quad$ Federal state budgetary institution

Makitov V.S. "High-Mountain geophysical institute», Nalchik, Russian Federation ljk_62@rambler.ru

\section{INFLUENCE THE DEFORMATION OF THE DROPS GROWING IN THE ELECTROMAGNETIC FIELD ON THEIR SCATTERING PROPERTIES}

Introduction. It is known that under conditions of free fall in clouds and precipitation the drops deform and take different forms. Experimental work carried out in the wind tunnel shows that the drops under the influence of the electromagnetic field deform even more. Such works allow to more accurately estimate the size, shape and orientation of the drops, which are used in the study of their scattering properties (backscattering cross sections).

Materials and methods of research.

To determine the shape factor of drops growing in thunderstorm clouds, the results of numerical modeling, laboratory studies in a wind tunnel and field experiments with a polarimeter were used. In the case of spherical drops, the scattering characteristics were calculated by Mie. For deformed drops, a variable separation method modified for particles of free spheroid shape was used.

Research results and discussion.

Calculations of the backscattering cross-section of deformed drops growing under the influence of a horizontal electric field are carried out. Drops with different degrees of deformation are considered. For comparative analysis, the calculations of the backscattering cross-section of spherical droplets are presented. It is shown that with an increase in the degree of deformation of drops growing in the electric field, the values of backscattering cross sections deviate more significantly from similar values for equivalent spheres.

Summary. The obtained results can be useful in the development of methods of thunderstorm clouds' radar research.

Keyword: $\quad$ scattering theory, backscattering cross section, the degree of drops' deformation, drops' spraying, thunderstorm clouds.

\section{ВВЕДЕНИЕ}

Разработка новых методов радиолокационньх исследований микрофизических параметров грозоградовых облаков предполагает учет влияния электрической активности облачной среды на процессы роста капель и кристаллов. В особенной степени это важно для правильной физической 
интерпретации характеристик отраженного радиолокационного сигнала при формировании ливневых осадков и града. Как известно, рост капель в электромагнитном поле сопровождается целым рядом физических явлений, детальный учет каждого из которых не всегда представляется возможным. В этом плане большой интерес представляют экспериментальные работы, выполненные в последние годы, авторы которых анализируют рост капель в аэродинамической трубе под влиянием электромагнитного поля с заданными характеристиками $[1,2]$.

В этих публикациях приводятся данные фоторегистрации растущих капель, выполненные с высоким пространственно-временным разрешением. Такой подход позволяет более точно оценить размеры, форму и ориентацию растущих в электромагнитном поле капель. Появляется уникальная возможность рассчитать рассеивающие характеристики отдельных частиц и радиолокационные параметры спектра растущих капель с учетом их реальных геометрических размеров. Очевидно, что для оценки максимальных значений радиолокационной отражаемости облачного объема, наиболее важной стадией роста капли является ее деформация перед финальным разбрызгиванием. Именно на этой стадии поперечное сечение обратного рассеяния капли достигает своего максимального значения. Исходя из того, что геометрические размеры растущих в электрическом поле капель могут значительно превосходить их максимальные значения в отсутствии поля, учет влияния этого фактора крайне важен при разработке радиолокационных методов количественной параметризации ливневых осадков и града, особенно в случае их одновременного выпадения [3]. Расчет поперечного сечения обратного рассеяния капли, растущей в электромагнитном поле на стадии, когда ее форма близка к сферической, а также в период максимальной деформации, когда ее форма приближается к сплющенному сфероиду, могут в значительной степени прояснить вклад каждого из этих значений в суммарную отражаемость облачного объема. В связи с этим целью настоящей работы является:

разработка методики расчета поперечного сечения обратного рассеяния для капель, имеющих форму произвольного сфероида и экстремальные размеры, характерные для режима роста под влиянием горизонтального электрического поля;

оценка влияния горизонтального электрического поля на рассевающие свойства капель на стадии достижения максимального размера перед разбрызгиванием. 


\section{МАТЕРИАЛЫ И МЕТОДЫ ИССЛЕДОВАНИЯ}

Дистанционные методы исследования дисперсных сред (облака, осадки, аэрозоли, межзведная пыль и т.д.) широко используемые в настоящее время в различных областях науки и имеют большое прикладное значение. В основе этих методов лежит точное решение задачи рассеяния электромагнитной волны изолированной сферической частищей, предложенное Ми. Это решение было получено на сферическом базисе методом разделения переменных. Выражение для расчета поперечников обратного рассеяния имеет вид [4]:

$$
\sigma(r, m, \lambda)=\frac{\lambda^{2}}{4 \pi}\left|\sum_{n=1}^{\infty}(-1)^{n}(2 n+1)\left(a_{n}-b_{n}\right)\right|^{2}
$$

$\begin{array}{ll}\text { где } & r- \\ & \gamma- \\ & m- \\ & a_{n} \text { и } b_{n}-\end{array}$

радиус частицы;

длина волны электромагнитного излучения;

комплексный показатель преломления;

коэффициенты, зависящие от цилиндрических функций Бесселя первого рода для решения внутри сферы и Ханкеля второго рода для решения вне сферы.

Однако реальные рассеиватели, как правило, являются несферическими, а теория Ми до сих пор должным образом не была развита для несферических частиц. Были известны лишь приближенные методы, область применения которых весьма ограничена. Сотрудники Санкт-Петербургского университета, решая задачу на сфероидальном базисе, модифицировали метод разделения переменных для частиц формы произвольного сфероида.

Согласно предложенному методу [5, 6], сфероидальная система координат вводится таким образом, что начало координат совпадает с центром сфероида. При этом большая ось сфероида обозначается через $a$, а малая через $b$. Предполагается, что на частицу падает плоская поляризованная волна, которая может быть представлена в виде суперпозиции вертикально поляризованной (ТМ мода) и горизонтально поляризованной (ТЕ мода) волн. Для каждой из этих волн электрический и магнитный вектора, соответственно вибрируют перпендикулярно плоскости падения. В этом случае выражения для поперечных сечений обратного рассеяния определяются следующим образом [6]:

для ТМ моды:

$$
\begin{aligned}
& \sigma^{T M}=\frac{4 \pi}{(2 \pi / \lambda)^{2}} \mid \sum_{l=1}^{\infty} i^{l} b_{l}^{(1)} S_{1 l}(c, \cos \alpha)-\sum_{m=1}^{\infty} \sum_{l=m}^{\infty} i^{(l-1)}\left(k_{1} a_{m l}^{(1)} S_{m l}(c, \cos \alpha)-\right. \\
& \left.-i b_{m l}^{(1)} \frac{d S_{1 l}(c, \cos \alpha)}{d \cos \alpha}\right)\left.\sin \alpha\right|^{2}
\end{aligned}
$$




$$
b_{l}^{(1)}=-2 i^{l} \frac{\left[\left(\xi_{0}^{2}-1\right)^{1 / 2} R_{1 l}^{(1)}\left(c, \xi_{0}\right)\right]}{\left[\left(\xi_{0}^{2}-1\right)^{1 / 2} R_{1 l}^{(3)}\left(c, \xi_{0}\right)\right]} N_{1 l}^{-2}(c) S_{1 l}(c, \cos \alpha)
$$

для ТЕ моды:

$$
\begin{aligned}
& \sigma^{T E}=\frac{4 \pi}{(2 \pi / \lambda)^{2}} \mid \sum_{l=1}^{\infty} i^{l} a_{l}^{(1)} S_{1 l}(c, \cos \alpha)-\sum_{m=1}^{\infty} \sum_{l=m}^{\infty} i^{(l-1)}\left(k_{1} a_{m l}^{(1)} S_{m l}(c, \cos \alpha)-\right. \\
& \left.-i b_{m l}^{(1)} \frac{d S_{1 l}(c, \cos \alpha)}{d \cos \alpha}\right)\left.\sin \alpha\right|^{2},
\end{aligned}
$$

$$
a_{l}^{(1)}=-2 i^{l} \frac{R_{1 l}^{(1)}\left(c, \xi_{0}\right)}{R_{1 l}^{(3)}\left(c, \xi_{0}\right)} N_{1 l}^{-2}(c) S_{1 l}(c, \cos \alpha),
$$

$\xi_{0}=\left(\frac{a}{b}\right)^{(1+\tilde{f}) / 2}\left[\left(\frac{a}{b}\right)-1\right]^{-1 / 2}$,

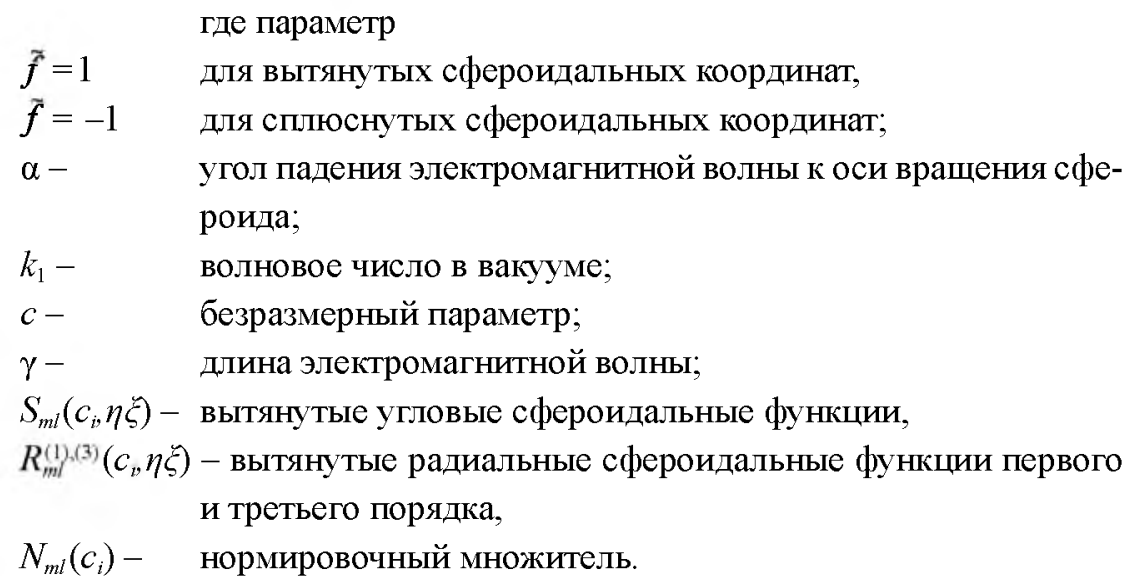

На практике для определения фактора формы капель, растущих в грозоградовых облаках, можно использовать выражение [7]:

$$
\frac{a}{b}=\frac{1}{1.012-0.144 \cdot\left(D_{v}\right)-1.03 \cdot\left(D_{v}\right)^{2}}
$$

где $D_{v}-\quad$ диаметр эквивалентной сферической капли в [cм].

Эта формула была получена путем обобщения результатов лабораторньх исследований в аэродинамической трубе, натурных экспериментов с использованием поляриметра и численного моделирования фактора формы капель 


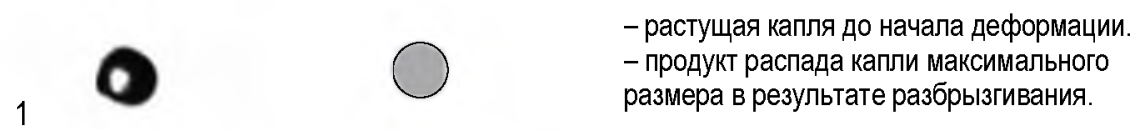

растущая капля при десормации в
горизонтальном электрическом поле
напряженностью $\mathrm{E}=500 \mathrm{kVm}^{-1}$
$\Delta t=23 \mathrm{~ms}$

2

растущая капля при деформации в
горизонтальном электрическом поле
напряженностью $\mathrm{E}=500 \mathrm{kVm}^{-1}$
$\Delta T=0 \mathrm{~ms}$

Рис. 1.

Приближенная схема представления растущей в электрическом поле капли с эквивалентным радиусом 3.625 мм.

Fig. 1. The approximate scheme representing growing in the electrical field droplet with equivalent radius $3.625 \mathrm{~mm}$.

Результаты, полученные в $[1,2]$ представляют повышенный интерес именно тем, что авторы анализируют рост капель в аэродинамической трубе под влиянием электрического поля заданной напряженности. На рисунке 1 размещена приближенная схема деформации капли, эквивалентный радиус которой равен 3.625 мм [1]. Слева показаны снимки деформируемой капли в различные моменты времени, а в центре приведены соответствующие геометрические формы, которыми аппроксимируются капли. На фрагменте 1 представлена капля, растущая при отсутствии электрического поля, ее форма близка к сферической. В этом случае аппроксимация формы капли сферой выглядит вполне оправданной. Также сферой можно аппроксимировать продукты распада капли максимального диаметра в результате разбрызгивания. Дальнейший рост капли, и влияние аэродинамических эффектов вызывают процесс деформации, и с момента $\Delta \tau=0$ форма капли аппроксимируется сфероидом (фрагмент 2). Именно с этого момента, на растущую в аэродинамической трубе каплю начинает действовать электрическое поле напряженностью $\mathrm{H}=500 \mathrm{\kappa BT} \cdot \mathrm{M}^{-1}$, под влиянием которого ее горизонтальные размеры заметно увеличиваются (по сравнению со случаем без электрического поля). При $\Delta \tau=23$ мс большая ось сфероида приближается к 20 мм (фрагмент $3)$, а при $\Delta \tau=59 . м c$ уже существенно превосходит это значение (фрагмент 4). В этот момент наиболее подходящей аппроксимацией формы растущей капли является сочетание двух сфероидов. 


\section{РЕЗУЛЬТАТЫ ИССЛЕДОВАНИЙ И ИХ ОБСУЖДЕНИЕ}

Как известно, в условиях свободного падения капли принимают различные формы. В период первоначального роста они сферические. По мере роста в облаках под действием сил гравитации и сопротивления воздуха форма капель меняется. Так, у капель более 1 мм нижняя часть начинает прогибаться внутрь; чем больше диаметр, тем больше прогибается основание. На определенном этапе капли начинают захватывать небольшой объем воздуха перед собой, приобретая форму сплющенного сфероида. Начиная, с диаметра $6 . м$ и более становятся неустойчивыми и начинают распадаться на отдельные фрагменты, размеры которых соответствуют размерам дождевых капель, которые и достигают поверхности земли. Как известно, в реальных грозоградовых облаках на рост капель оказывает влияние еще и электрическое поле, инициируемое естественной грозовой активностью, сопровождающей процесс формирования ливневых осадков и града. В этом случае форма, ориентация и размер могут существенно отличаться от аналогичных значений растущих капель при отсутствии электрического поля.

Рассматривались рассеивающие свойства капель, падающих в области осадков, где преобладают температуры около $10^{\circ} \mathrm{C}$. В этой части облака могут присутствовать крупные капли, диаметр которых может достигать 8 мM.

Характеристики рассеяния реальных капель определялись для различных размеров и форм.

Расчеты поперечных сечений обратного рассеяния сферических капель были проведены по Ми, а сфероидальных капель по В.Г. Фарафонову с учетом фактора формы. Поперечные сечения обратного рассеяния при различной поляризации сигнала зависит от фактора формы (рис. 2). Чем больше размер капли, тем больше разница между поперечниками обратного рассеяния вертикальной (ТМ - моды) и горизонтальной (ТЕ - моды) поляризации. До размеров $r<0.12 \mathrm{~cm}$ (рэлеевская область), три кривые совпадают. От размеров $r<0.12 \mathrm{~cm}$ проявляется влияние формы частицы на поперечники обратного рассеяния [8].

В таблице 1 приводятся результаты расчетов нормализованных поперечных сечений обратного рассеяния (отнесенные к $\pi R_{v}^{2}$ ) для деформированных капель, позаимствованных из работы [1] при наличии горизонтального электрического поля. В расчетах длина волны была равна $\lambda=5.5 \mathrm{~cm}$, что соответствует длине волны электромагнитного излучения доплеровского метеорологического радиолокатора с двойной поляризацией ДМРЛ-С.

Графическая интерпретация результатов приводится на рисунке 3 , на котором показаны зависимости нормализованных поперечных сечений обратного рассеяния от эквивалентного радиуса для случаев с различной степенью деформации капель с учетом влияния горизонтального электрического поля и без. 

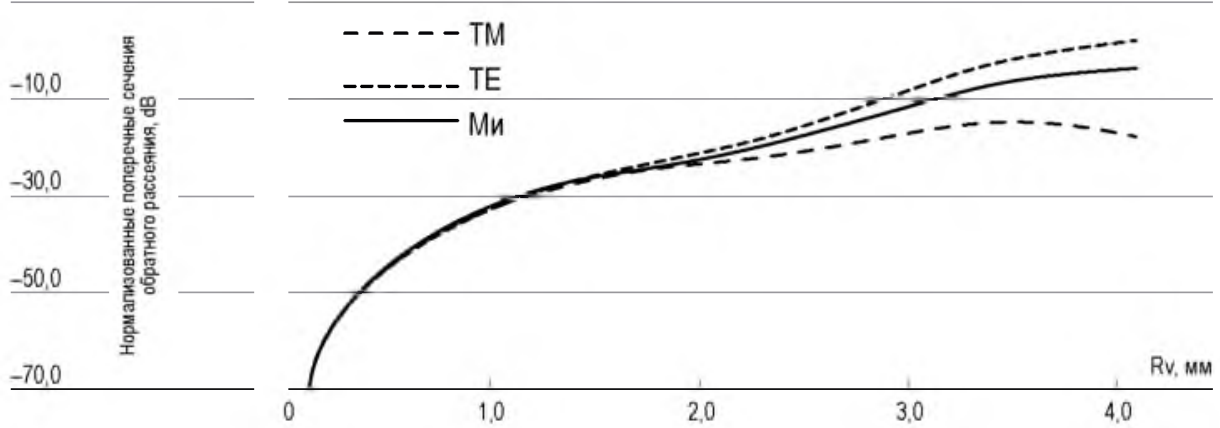

Pис. 2.

Зависимость нормализованных сечений обратного рассеяния капель воды от эквивалентного радиуса для длины волны $\lambda=5.5 \mathrm{cm:} \mathrm{Mu}$ - эквивалентная сферическая капля, TM - горизонтально и ТЕ - вертикально поляризованные составляющие для сфероидальной капли.

Fig. 2. Dependence of normalized backscattering cross sections of water drop on the equivalent radius for wavelength $\lambda=5.5 \mathrm{~cm}: \mathrm{Mu}$ - an equivalent spherical drop, TM - horizontal and TE - vertically polarized component for the spheroidal drop.

ТабЛИца 1. НОРМАЛИЗОВАННЫЕ ПОПЕРЕЧНЫЕ СЕЧЕНИЯ ОБРАТНОГО РАССЕЯНИЯ КАПЕЛЬ С РАЗЛИЧНОЙ СТЕПЕНЬЮ ДЕФОРМАЦИИ ПРИ НАЛИЧИИ ГОРИЗОНТАЛЬНОГО ЭЛЕКТРИЧЕСКОГО ПОЛЯ

Table 1. Normalized backscattering cross sections of drops with varying degrees of deformation in the presence of a horizontal electric field

\begin{tabular}{|c|c|c|c|c|c|c|}
\hline \multirow[t]{2}{*}{$\begin{array}{l}\text { Эквивалентный } \\
\text { радиус, мМ }\end{array}$} & \multicolumn{2}{|c|}{ Оси сфероида } & \multirow{2}{*}{$\begin{array}{l}\text { Фактор } \\
\text { формы, } \\
\text { a/b }\end{array}$} & \multicolumn{3}{|c|}{$\begin{array}{l}\text { Нормализованные поперечные } \\
\text { сечения обратного рассеяния, dB }\end{array}$} \\
\hline & a & b & & $\begin{array}{l}\text { сффероид, } \\
\text { TM }\end{array}$ & $\begin{array}{l}\text { сффероид, } \\
\text { TE }\end{array}$ & $\begin{array}{l}\text { copepa } \\
\text { Mu }\end{array}$ \\
\hline \multicolumn{7}{|c|}{ Средняя деформация капель } \\
\hline 3,3 & 14,98 & 4,39 & 3,4123 & $-20,82$ & $-1,37$ & $-7,39$ \\
\hline 3,5 & 15,8 & 4,67 & 3,3833 & $-19,58$ & $-0,5$ & $-6,04$ \\
\hline 3,625 & 17,08 & 4,73 & 3,6110 & $-19,3$ & 0,27 & $-5,4$ \\
\hline \multicolumn{7}{|c|}{ Максимальная деформация капель } \\
\hline 3,3 & 17,7 & 4,04 & 4,3812 & $-21,46$ & 0,06 & $-7,39$ \\
\hline 3,5 & 19,05 & 4,25 & 4,4824 & $-20,96$ & 1,08 & $-6,04$ \\
\hline 3,625 & 20,07 & 4,36 & 4,6032 & $-20,68$ & 1,63 & $-5,4$ \\
\hline \multicolumn{7}{|c|}{ Экстремальная деформация капель } \\
\hline 3,5 & 29 & 3,44 & 8,4302 & $-21,7$ & 4,81 & $-6,04$ \\
\hline 3,625 & 42 & 3,01 & 13,9535 & $-22,50$ & 7,68 & $-5,4$ \\
\hline
\end{tabular}



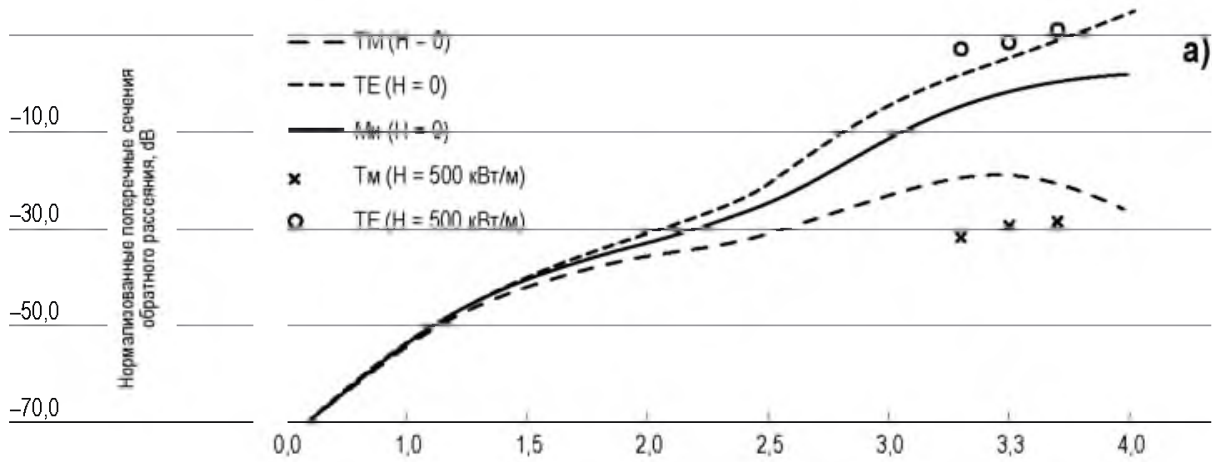

b)
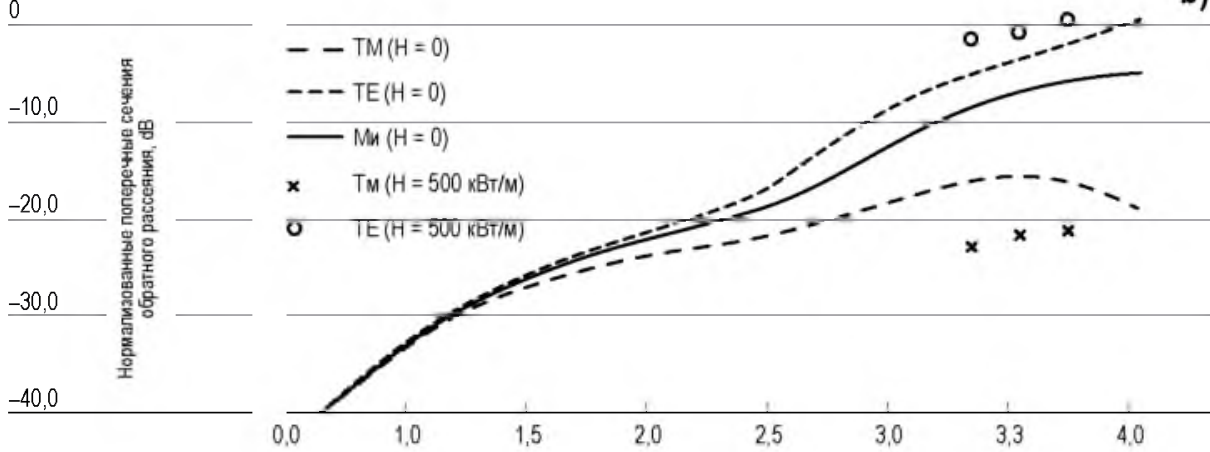

c)
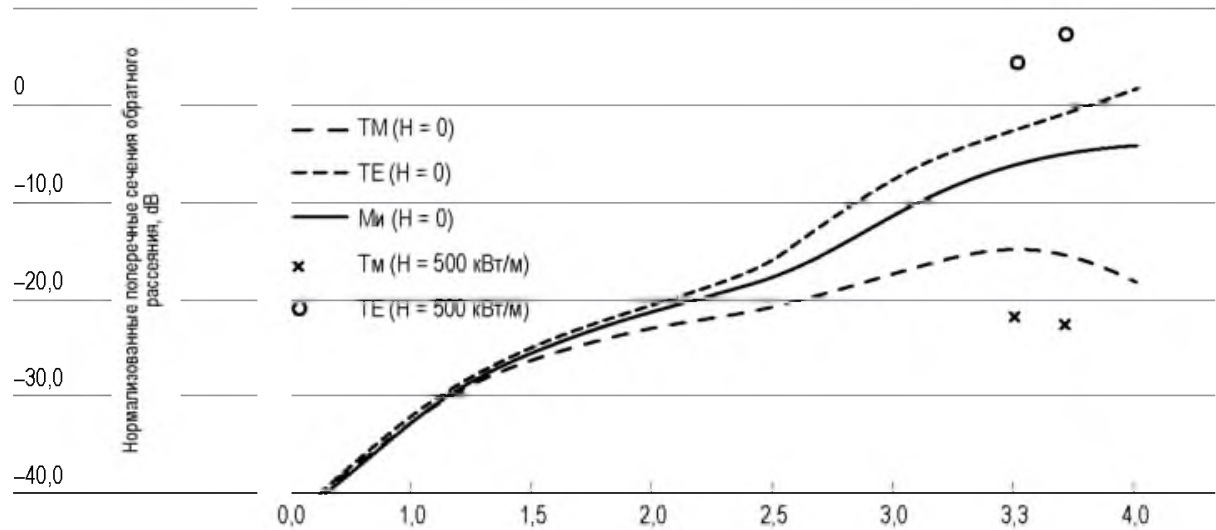

Рис. 3. Нормализованные поперечные сечения обратного рассеяния капель с учетом влияния горизонтального электрического поля для случаев с различной степенью деформации: a) средней, б) максимальной, с) экстремальной.

Fig. 3. Normalized backscattering cross sections of droplets taking into account the influence of the horizontal electric field for cases with different degrees of deformation: a) average, b) maximum, c) extreme. 
Сопоставительный анализ рисунков 2 и 3 показывает, что при увеличении степени деформации растущих в электрическом поле капель, значения поперечных сечений обратного рассеяния более существенно отклоняются от аналогичных значений для эквивалентных сфер. При этом различия между поперечниками обратного рассеяния вертикальной (ТМ - моды) и горизонтальной (ТЕ - моды) поляризации для случаев средней (рис. 3 а) и максимальной (рис. 3 б) деформации практически совпадают, хотя и значительно отличаются от значений для частиц с идентичным эквивалентным радиусом при отсутствии электрического поля (рис. 1). Мы имеем два случая с экстремальной деформацией, когда горизонтальные размеры капель перед разбрызгиванием достигали 2.9 и $4.2 \mathrm{mм}$ соответственно (рис. 3 с). Очевидно, что в естественных условиях, при отсутствии электрического поля приближение растущей капли к таким размерам невозможно. Именно для этих размеров отмечается максимальное различие между горизонтально и вертикально поляризованными составляющими. Как известно, значение реальной радиолокационной отражаемости определяются спектральными характеристиками облачного объема, которые в большей степени зависят именно от наиболее крупных частиц спектра. В этом плане наличие таких капель может существенно изменить значения, как радиолокационной отражаемости, так и поляризационных характеристик зон локализации ливневых осадков и града.

\section{Выводы}

Предложенная в работе методика расчета поперечного сечения обратного рассеяния капли воды позволяет рассчитывать горизонтальную и вертикальную поляризационные составляющие для частиц с любой степенью деформации. Для расчета поперечного сечения обратного рассеяния частиц различного размера и формы были использованы экспериментальные данные для капель, растущих в аэродинамической трубе под влиянием горизонтального электрического поля.

Показано, что при увеличении степени деформации растущей в электрическом поле капли, значения поперечного сечения обратного рассеяния более сушественно отклоняются от аналогичных значений для эквивалентных сфер воды. Различия между поперечными сечениями обратного рассеяния при вертикальной и горизонтальной поляризации для случаев средней и максимальной деформации практически совпадают, хотя и значительно отличаются от значений для частиц с идентичным эквивалентным радиусом при отсутствии электрического поля. Максимальное различие между горизонтально и вертикально поляризованными составляющими отмечается для капель формы сфероида с экстремальными горизонтальными размерами (2.9 и $4.2 \mathrm{MM})$.

Полученные результаты могут быть полезны при разработке методов радиолокационного исследования грозоградовых облаков. В том числе с ис- 
пользованием ДМРЛ-С (доплеровский метеорологический радиолокатор с двойной поляризацией), которые устанавливаются Росгидрометом на территории России с целью создания единой системы радиолокационных метеорологических наблюдений.

\section{БИБЛИОГРАФИЧЕСКИЙ СПИСОК}

1. Bhalwankar Rohini, C. G. Deshpande, A. K. Kamra. Breakup modes of the drops suspended in a vertical wind tunnel in presence of the horizontal electric field // Journal of Geophysical Research: Atmospheres. 10.1002/2016 JD025805. p. 1838-1849.

2. Bhalwankar R. V., Kamra A. K. A wind tunnel investigation of the deformation of water drops in the vertical and horizontal electric fields // Journal of Geophysical Research: Atmospheres. 2007. Vol. 112, D10215. doi:10.1029/2006JD007863.

3. Makitov Viktor. Radar measurements of integral parameters of hailstorms used on hail suppression projects // Journal of Atmospheric Research. 2007. Vol. 83. p. 380-388.

4. Bohren C. and Huffman D. Absorption and Scattering of Light by Small Particles, New York: John Wiley \& Sons, 1983. p. 660.

5. http://www.astro.spbu. ru (дата обращения 08.01.2019).

6. Farafonov V.G. Application of non-orthogonal bases in the theory of light scattering by spheroidal particles, In Kokhanovsky A.A. (Ed.), Light Scattering Reviews, London: Springer-Praxis. 2013. №8. p. 189-268

7. Andsager K., Beard K., and Laird N. (1999). Laboratory measurements of axis ratios for large raindrops // J. Atmos. Sci. 56. p. 2673 2683

8. Кагермазов А.Х., Созаева Л.Т. Расчет поперечных сечений обратного рассеяния несферических капель в облаках и осадках // Доклады Адыгской (Черкесской) Международной академии наук. 2014. Т. 16. № 4. С. 91-96.

\section{REFERENCES}

1. Bhalwankar Rohini, C. G. Deshpande, A. K. Kamra. Breakup modes of the drops suspended in a vertical wind tunnel in presence of the horizontal electric field // Journal of Geophysical Research: Atmospheres. 10.1002/2016 JD025805. p. 1838-1849.

2. Bhalwankar R. V., Kamra A.K.A wind tunnel investigation of the deformation of water drops in the vertical and horizontal electric fields // Journal of Geophysical Research: Atmospheres. 2007. Vol. 112, D10215. doi:10.1029/2006JD007863.

3. Makitov Viktor. Radar measurements of integral parameters of hailstorms used on hail suppression projects / Journal of Atmospheric Research. 2007. Vol. 83. p. 380-388. 
4. Bohren C. and Huffman D. Absorption and Scattering of Light by Small Particles, New York: John Wiley \& Sons, 1983. . p. 660.

5. http://www.astro. spbu.ru (дата обращения 08.01.2019).

6. Farafonov V.G. Application of non-orthogonal bases in the theory of light scattering by spheroidal particles, In Kokhanovsky A.A. (Ed.), Light Scattering Reviews, London: Springer-Praxis. 2013. №8. p. 189-268

7. Andsager K., Beard K., and Laird N. (1999). Laboratory measurements of axis ratios for large raindrops // J. Atmos. Sci. 56. p. 2673-2683.

8. Kagermazov A.H., Sozaeva L.T., Calculation of back scattering cross section of aspheric drops in clouds and precipitation, Reports of the Adyghe (Circassian) International academy of Sciences, 16 (2014), 91-96.

Рукопись поступила в редакцию 13.08.2019, принята к публикации 02.09.2019.

\section{СВЕДЕНИЯ ОБ АВТОРАХ}

Созаева Лежинка Танашевна - кандидат физико-математических наук, старший научный сотрудник, ФГБУ «Высокогорный геофизический институт»

Адрес: РФ, Кабардино-Балкарская Республика, г. Нальчик, пр. Ленина, 2. Scopus ID 57204527832. ljk_62@rambler.ru. +7 (8 928) 7232008

Макитов Виктор Сафарович - кандидат физико-математических наук, старший научный сотрудник, ФГБУ «Высокогорный геофизический институт»

Адрес: РФ, Кабардино-Балкарская Республика, г. Нальчик, пр. Ленина, 2. makitov_v@mail.ru. +7 (8662) 402484

\section{About the authors}

Sozaeva Lezhinka Tanashevna - candidate of physical and mathematical Sciences, senior research associate of department of physics of clouds of Federal state budgetary institution «High-Mountain Geophysical Institute»

Address: Russia, Kabardino-Balkar Republic, Nalchik, Lenin Ave., 2. Scopus ID 57204527832. ljk_62@rambler.ru. +7 (8 928) 7232008

Makitov Viktor Safarovich - candidate of physical and mathematical Sciences, senior research associate of department of physics of clouds of Federal state budgetary institution «High-Mountain Geophysical Institute»

Address: Russia, Kabardino-Balkar Republic, Nalchik, Lenin Ave., 2. makitov_v@mail.ru. +7 (8662) 402484. 